\title{
Is the Currently Used Prescription Adjudication Date a Good Proxy for Calculating Medication Refill Adherence?
}

\author{
Corey A. Lester, PharmD, MS; Kevin A. Look, PharmD, PhD; and Michelle A. Chui, PharmD, PhD
}

\begin{abstract}
BACKGROUND: The Centers for Medicare \& Medicaid Services (CMS) adopted the proportion of days covered (PDC) calculation for use in their Five-Star Quality Rating System for Medicare Advantage and Prescription Drug Plans. This calculation uses the prescription adjudication date (i.e., date the prescription is billed to the benefits manager by a pharmacy) as a proxy for medication adherence. Adherence programs, such as automatic refill programs, have become commonplace in community pharmacy and have been identified by industry leaders as interfering with the ability to accurately measure adherence using PDC.
\end{abstract}

OBJECTIVE: To evaluate the prescription pickup date instead of the currently used adjudication date to calculate PDC in the presence of a community pharmacy automatic refill program.

METHODS: This study used a post-only quasi-experimental design with patients aged 65 years or older enrolled in automatic and manual refill programs in a 29-store community pharmacy chain during 2014. PDC was calculated using the prescription adjudication date and pickup date (i.e., date the patient brought the medication home) using pharmacy dispensing data for CMS adherence metrics medications, including statins, renin angiotensin aldosterone system antagonists (RASA), and noninsulin diabetes medications. Mann-Whitney $U$ and effect size calculations evaluated differences in PDC between automatic and manual refill prescriptions for the adjudication date and pickup date, as well as the difference in PDC between adjudication and pickup date.

RESULTS: 10,936 prescriptions were included with $21.9 \%$ enrolled in the automatic refill program. Mean (SD) adherence was 88.6 (17.6) and 86.4 (17.1) for automatic refills and 85.8 (19.0) and 85.0 (18.9) for manual refills, using the adjudication date and pickup date PDC, respectively. Significant difference existed between automatic and manual refill prescriptions using the adjudication date $(P<0.001)$ but not for the pickup date. The difference between adjudication and pickup date PDC ranged from $0 \%$ to $32.0 \%$ for automatic refills and $0 \%$ to $38.7 \%$ for manual refills. The difference between adjudication and pickup date PDC was significant when comparing automatic and manual refill prescriptions $(P<0.001)$.

CONCLUSIONS: The artificial inflation seen with adjudication date PDC indicates that the prescription pickup date is a more accurate reflection of patient medication taking. Automatic refills resulted in a less reliable PDC compared with manual refill prescriptions. Discussion about the continued use of the adjudication date to calculate PDC is needed.

J Manag Care Spec Pharm. 2016;22(11):1311-17

Copyright @ 2016, Academy of Managed Care Pharmacy. All rights reserved.

\section{What is already known about this subject}

The prescription adjudication date is currently used to measure the proportion of days covered (PDC) adherence metric by researchers across the country and by the Centers for Medicare $\leftleftarrows$ Medicaid Services in their Five-Star Quality Rating System The number of days between the prescription fill and prescription pickup date is significantly larger for automatic refills compared with manual refills.

Automatic prescription refill programs have become commonplace in the community pharmacy industry over the last several years.

\section{What this study adds}

This study considers the use of the prescription pickup date as the variable to use when calculating PDC and how an automatic prescription refill program influences the PDC calculation.

Significant differences in calculated adherence for the adjudication date PDC and pickup date PDC exist with an automatic refill program.

This study highlights an important consideration to improve the validity and reliability of the PDC adherence measure.

T he Centers for Medicare \& Medicaid Services (CMS) adopted the proportion of days covered (PDC) methodology to measure medication adherence in their Five-Star Quality Rating System. ${ }^{1}$ These adherence metrics contribute to enrollment decisions by beneficiaries and, in part, determine which plans are eligible for bonus payments that totaled \$3.1 billion in 2012., ${ }^{2,3}$ As a result, CMS has recognized the need for scrutiny of these metrics methodologies to ensure the integrity of the star program. ${ }^{4}$

Variations in the methods used to calculate medication adherence can result in significant changes to patient refill adherence rates when using prescription drug claims data. Sources of variation include incorporating a run-in period, changing inclusion and exclusion criteria, and accounting for overlapping days supply between refills..$^{5-7}$ Previous studies assessing medication refill adherence have exclusively relied on the prescription adjudication date (i.e., the date the prescription is billed to a health plan) to indicate when a prescription medication was obtained by a patient..$^{7-9}$ However, our previous research suggests that the prescription adjudication date is often not the same date that the prescription is picked up by the patient..$^{8-10}$ Our findings also suggest a mean pickup lag 
of approximately 7 days when a prescription is filled using an automatic prescription refill program.

Automatic prescription refill programs have been widely adopted in community pharmacies and are promoted as a convenient way for patients to refill their medications. ${ }^{11,12}$ These programs refill patients' chronic medications on a standardized and recurrent basis up to 7 days before the previous refill runs out. The pharmacy-dispensing software uses an algorithm to determine which automatic refills will enter the queue for processing that day. For example, the next refill for a 30-day supply of medication would be automatically generated by the dispensing software 23 days after a patient picked up the previous fill. This is in contrast with the way prescriptions are traditionally filled, in which the patient manually requests the prescription in person or by telephone, internet, or mobile application.

A recently published study suggests that automatic refill programs improve adherence by $1.4 \%-3.0 \%$ when calculated using medication possession ratio (MPR). ${ }^{12}$ Community pharmacy executives have reported that these programs can inflate calculated patient refill adherence and quality rating metrics such as those used by CMS. The extent to which these programs influence medication refill adherence calculations is unknown. The purpose of this study is to evaluate the effect of using the prescription pickup date in place of the adjudication date to calculate medication adherence using PDC for automatic and manual refill prescriptions.

\section{Methods}

This study used a post-only quasi-experimental design with automatic and manual refill study groups. The study was approved by an institutional review board.

\section{Data Source}

Prescription-dispensing data were obtained from a 29-store community pharmacy chain in a midwestern state. The majority of these pharmacies are located in rural suburbs. The dispensing data were obtained for the 2014 calendar year, which is consistent with the approach that CMS uses to perform their adherence metric calculations.

\section{Sample Population}

The study sample included patients aged 65 years and older who were taking at least 1 of the following chronic medications used in the CMS star ratings adherence metrics: HMGCoA reductase inhibitors (i.e., statins), angiotensin-converting enzyme inhibitors, angiotensin-II receptor antagonists, sulfonylureas, biguanides, dipeptidyl peptidase IV inhibitors, thiazolidinediones, and subtype II sodium-glucose transport protein inhibitors. Patients had to have at least 2 prescription fills of that medication during the 2014 calendar year. To ensure that patients receiving 90 -day supplies of medication were able to obtain a second fill during the observation period, the first fill must have occurred at least 91 days before the end of the calendar year. These criteria were chosen to be consistent with CMS methodology. This resulted in 2 study groups: an automatic refill group and a manual refill group. Figure 1 shows a flowchart of the sample selection.

\section{Variables}

Prescription drug refill adherence was calculated using the PDC method. PDC was initially proposed in 2002 as a new method to calculate prescription refill adherence using prescription claims data. ${ }^{13}$ PDC is considered to be an improvement over alternative approaches such as MPR because it accounts for medication persistence (i.e., continuing to take a medication over a specified treatment period). ${ }^{14}$ This means that PDC is less likely to result in higher refill adherence rates because it considers patients who have stopped taking their medication during the observation period. PDC is also more consistently defined when compared with MPR; however, variations of this calculation still exist, such as accounting for concurrent medication use and inclusion of a run-in period. ${ }^{5}$ In light of these factors, we employed the criteria used by CMS, which is based on the approach developed by the Pharmacy Quality Alliance. ${ }^{6,15}$ However, an important difference that was not performed in this study was the adjustment of the PDC calculation by CMS to exclude days in which a patient is admitted to the hospital, long-term care facility, or deceased..$^{15}$ The PDC calculation for this study was determined using the following equation:

$$
\text { PDC }=\frac{\text { Number of days covered with medication }}{\text { Number of days in observation period }}
$$

The numerator (number of days covered) was based on the prescription date used (i.e., adjudication or pickup date) plus the days supply for each fill. The days supply was defined as the value entered by the pharmacy that corresponded to the number of days that the medication fill would last based on the written directions. The adjudication date was defined as the date that the prescription claim was submitted to the pharmacy benefits manager for payment. The prescription pickup date was defined as the date that the patient picked up the prescription from the pharmacy. This date is documented and stored electronically by the pharmacy chain for the purposes of audits from pharmacy benefits managers and other third parties. In order to maintain a meaningful comparison between the adjudication date compared with the pickup date, the number of days in the observation period was fixed. This means that when calculating PDC with the adjudication date and the pickup date, the denominator of the PDC formula was always based on the adjudication date for each prescription. 


\section{FIGURE 1 Sample Selection Flowchart}

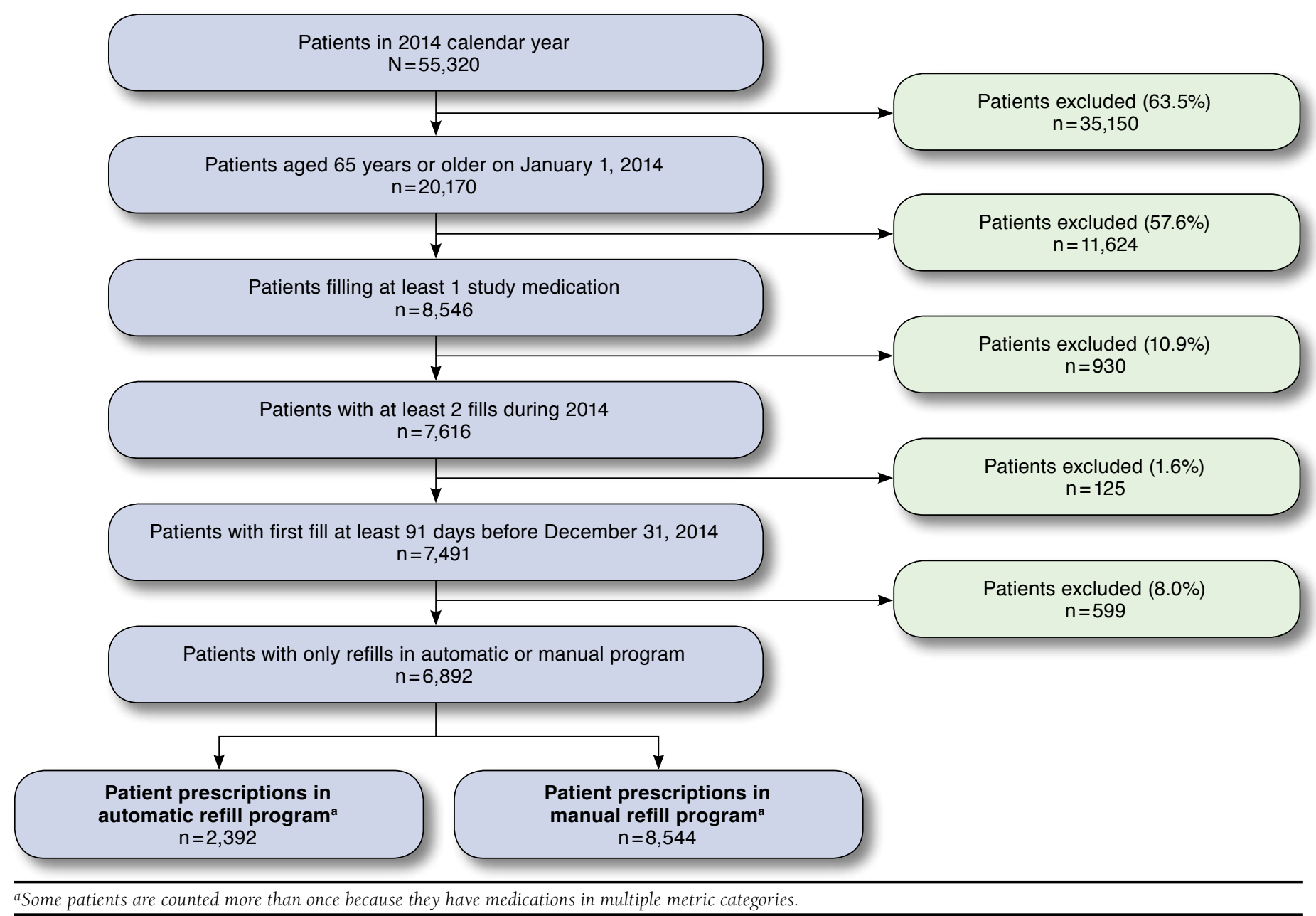

To account for the possibility that a pharmacy used multiple National Drug Code (NDC) numbers for the same medication, the Generic Product Identifier (GPI), made available by the Pharmacy Quality Alliance, was used. GPI aggregated NDC numbers to the generic product name. PDC accounts for overlapping days supply by adjusting the start date of the next fill to be equal to the previous fill plus the days supply. This adjustment also occurred when patients switched between 2 NDC numbers of the same generic medication. The taking of more than 1 medication in a single adherence metric (e.g., multiple diabetes medications) did not result in an adjustment of the patient's start date, since the patient likely took those medications concurrently. To be considered a covered day, however, patients only needed to have 1 of the medications available to them based on the prescription date being used in the calculation.
Medication adherence was calculated using 2 different PDC approaches-adjudication date and pickup date. The difference between the adjudication date PDC and the pickup date PDC was also determined for both of the refill groups by taking the PDC value calculated using the pickup date and subtracting it from the PDC value calculated using the adjudication date.

Independent variables were age, gender, and number of chronic medications. Birth dates were converted to age by subtracting patients' dates of birth from January 1, 2014, to find ages at the beginning of 2014. To determine a proxy for the number of chronic medications, the number of unique NDC numbers were identified using the same inclusion criteria already specified for the adherence metrics. A GPI was not available for all medications prescribed, so it is possible that the number of chronic medications reported is an overestimation, since patients switching between 2 NDC numbers for the same generic medication counted as 2 chronic medications. 
Is the Currently Used Prescription Adjudication Date a Good Proxy for Calculating Medication Refill Adherence?

TABLE 1 Prescription Demographics by Refill Type

\begin{tabular}{l|c|c}
\hline \multirow{2}{*}{} & \multicolumn{2}{|c}{ Refill Type } \\
\cline { 2 - 3 } & Automatic & Manual \\
\hline Number of prescriptions & 2,392 & 8,544 \\
\hline Gender & $1,218(50.9)^{\mathrm{a}}$ & $4,178(48.9)^{\mathrm{a}}$ \\
\hline Male, $\mathrm{n}(\%)$ & $79.4 \pm 10.1^{\mathrm{b}}$ & $79.7 \pm 9.8^{\mathrm{b}}$ \\
\hline Mean age $\pm S D$ & $8.7 \pm 5.3^{\mathrm{c}}$ & $8.0 \pm 5.6^{\mathrm{c}}$ \\
\hline $\begin{array}{l}\text { Mean number of NDC numbers } \\
\text { per patient } \pm \text { SD }\end{array}$ & \\
\hline${ }^{a}$ Chi-square test, $P<0.05$. & \\
${ }^{b}$ Mann-Whitney $U$ test, $P<0.01$. \\
${ }^{c}$ Mann-Whitney U test, $P<0.001$. \\
NDC=National Drug Code; $S D=$ standard deviation. \\
\hline
\end{tabular}

\section{Data Analysis}

Descriptive statistics were used to characterize the distribution of data and included the mean, median, and range of the data. Chi-square tests and Mann-Whitney $\mathrm{U}$ tests were performed to examine differences in corresponding demographics between the automatic refill and manual refill cohorts. The MannWhitney $\mathrm{U}$ test was also performed to determine if there were significant differences between the cohorts for each of the comparisons being examined. For significant findings, nonparametric effect size calculations using Cliff's delta and the Vargha and Delaney A measure were performed to measure the magnitude of the differences between the cohorts and accounts for influence of sample size. Romano et al. (2006) suggest Cliff's delta thresholds of negligible $(<0.147)$, small $(<0.33)$, medium $(<0.474)$, and large $(>0.474)$ be used to help interpret results. ${ }^{16}$ Nonparametric tests are considered more robust compared with parametric tests, since they do not make assumptions about the distribution of data and are not violated when data lack normality. ${ }^{17}$ All data cleaning, preprocessing, and statistical analysis was performed using R 3.2.1 (R Foundation for Statistical Computing, Vienna, Austria).

\section{Results}

Table 1 presents demographic results for the refill cohorts. There were 10,936 prescriptions analyzed; of those, $21.9 \%$ were filled using the automatic refill program. The mean age (standard deviation [SD]) of patients was 79.4 (10.1) years in the automatic refill group and 79.7 (9.8) years in the manual refill group. Patients enrolled in the automatic refill program were taking less than 1 additional chronic medication compared with the manual refill group. Statistically significant differences between the refill programs were found for gender and the number of chronic medications.

Table 2 presents descriptive statistics for prescription refill adherence rates. Using the adjudication date to calculate PDC resulted in mean refill adherence rates (SD) of 88.6\% (17.6) and $85.8 \%$ (19.0) for the automatic and manual refill programs,

\begin{tabular}{|c|c|c|c|}
\hline Measure & $\operatorname{Mean}(\%) \pm S D$ & Median (\%) & Range (\%) \\
\hline \multicolumn{4}{|c|}{ djudication date PDC } \\
\hline Automatic refill & $88.6 \pm 17.6$ & 96.6 & $5.2-100.0$ \\
\hline Manual refill & $85.8 \pm 19.0$ & 94.7 & $5.2-100.0$ \\
\hline \multicolumn{4}{|l|}{ ickup date PDC } \\
\hline Automatic refill & $86.4 \pm 17.1$ & 93.6 & $5.2-100.0$ \\
\hline Manual refill & $85.0 \pm 18.9$ & 93.6 & $5.2-100.0$ \\
\hline \multicolumn{4}{|l|}{ ifference in PDC } \\
\hline Automatic refill & $2.2 \pm 3.1$ & 1.4 & $0.0-32.0$ \\
\hline Manual refill & $0.7 \pm 2.4$ & 0.0 & $0.0-38.7$ \\
\hline
\end{tabular}

respectively. Using the pickup date to calculate PDC resulted in mean adherence rates of $86.4 \%$ (17.1) and $85.0 \%$ (18.9) for the automatic and manual refill groups. The range in adherence for adjudication date PDC and pickup date PDC was 5.2\%-100.0\% for automatic and manual refill groups. Of the prescriptions enrolled in the automatic refill program, $71.6 \%$ had a difference in PDC when using the adjudication date compared with the pickup date. This difference compares with $46.3 \%$ of prescriptions enrolled in the manual refill program. The mean differences (SD) between the adjudication date PDC and pickup date PDC were 2.2\% (3.1) and 0.7\% (2.4) for the automatic and manual refill groups, respectively.

Table 3 provides the results for each comparison. Significant differences in adjudication date PDC between the automatic and manual refill prescriptions were found $(P<0.001)$. The effect size for this comparison was negligible. There were no significant differences found between the automatic and manual refill group when calculating PDC using the pickup date. Finally, the difference between the adjudication date PDC and pickup date PDC was significant $(P<0.001)$, with a medium effect size. The Vargha and Delaney A measure for this comparison means that there is a $72 \%$ chance a randomly selected observation from the automatic refill group would have a greater difference in PDC than a randomly selected observation from the manual refill group.

\section{Discussion}

This is the first study to consider the prescription pickup date as a proxy for determining patient refill adherence. The findings may also explain the improvements in adherence from automatic refill programs found in a recently published study. ${ }^{12}$ The findings indicate that the prescription pickup date results in lower refill adherence rates when compared with the adjudication date. In addition, the negligible, but statistically significant, improvements in adherence seen with automatic refills compared with manual refills disappear when using the pickup date. The amount of difference between the adjudication date 
Is the Currently Used Prescription Adjudication Date a Good Proxy for Calculating Medication Refill Adherence?

TABLE 3 Comparison of Automatic Versus Manual Refills for Each PDC Calculation

\begin{tabular}{l|c|c|c|c|c|c}
\hline \multirow{2}{*}{ PDC Calculation } & \multicolumn{3}{|c|}{ Mann-Whitney U } & \multicolumn{2}{c}{ Vargha and } \\
Delaney A \\
\cline { 2 - 7 } & $\mathbf{U}$ & $\mathbf{9 5 \%}$ CI & P Value & Estimate & Size of Effect & Estimate \\
\hline Adjudication date PDC & 0.9 & $0.7-1.2$ & $<0.001$ & 0.13 & Negligible & 0.57 \\
\hline Pickup date PDC & -0.2 & $-0.5-0.03$ & 0.120 & N/A & N/A & N/A \\
\hline PDC difference (adjudication-pickup) & 1.0 & $0.9-1.1$ & $<0.001$ & 0.43 & Medium & 0.72 \\
\hline
\end{tabular}

Note: A positive difference indicates that refill adherence was higher with automatic prescription refills.

$C I=$ confidence interval; $N / A=$ not available; $P D C=$ proportion of days covered.

and pickup date is greater with automatic refills compared with manual refills. These findings raise important questions about the accuracy of the method for calculating PDC used by researchers and the CMS star rating program.

The adjudication date resulted in a higher PDC compared with the pickup date when the prescription was not picked up by the patient for some time. This result is especially important at the end of the calendar year. For example, consider a prescription that was adjudicated on December 1, 2014, for a 90-day supply, which the patient did not pick up until December 14, 2012-2 weeks after adjudication. Using the adjudication date, covered days would be from December 1, 2014, through December 31, 2014 (i.e., 31 covered days). Using the pickup date, however, the covered days would be from December 14, 2014, through December 31, 2014 (i.e., 18 covered days added to the numerator). There is a 13-day difference in medication coverage when comparing the 2 prescription dates for the same patient.

This difference means that health plans can get credit for covered days when a prescription is still in the pharmacy waiting to be picked up, which translates into higher medication adherence rates by using the adjudication date. In this study's findings, the automatic refill program showed what appears to be an artificial improvement in patient refill adherence compared with manual refill groups when using the adjudication date. This result is further evidenced by a higher mean adjudication date PDC in the automatic refill group compared with the manual refill group, which did not happen when using the pickup date PDC. This artificial inflation means that the automatic refill program was not changing the medication-taking behavior of patients; it only appeared to be doing so as a result of using the adjudication date to calculate PDC.

The rates and degree of differences in PDC between the adjudication and pickup date are increased with automatic refill programs. These larger and more frequent discrepancies indicate that the PDC calculation is less reliable when an automatic refill program is used. As a result, plans that have a greater proportion of patients enrolled in an automatic refill program at community pharmacies are benefiting from higher PDC rates, which translates into better adherence metrics using the adjudication date. It is important to note that this would not be an issue if all plans had an equal proportion of patients enrolled in an automatic refill program, since CMS uses data submitted by all of the plans to determine star rating cutpoints. Based on this study's findings, variations in the prevalence of patients enrolled in automatic refill programs at different health plans requires further investigation.

The high rates of adherence found in this study population may have resulted in a diminished influence of the automatic refill program on refill adherence. Many patients had PDCs close to $100 \%$, meaning that refilling a prescription sooner would not result in a greater number of covered days. Patients with a PDC of $80 \%$ or lower may have experienced greater improvement in adherence with the automatic refill program and adjudication date, since the time spent in the pharmacy would be greater, translating into more covered days. Further investigation of these effects for patients not adherent to their medications is warranted.

These findings demonstrate that while the adjudication date has traditionally been used to calculate PDC, the growing emphasis on developing programs that improve medication adherence quality ratings are contributing to less accurate adherence measurement and creating potentially inaccurate star ratings. This issue is important because CMS rewards Medicare Advantage plans with bonus payments for having an overall star rating of 4 or 5 stars. Adherence metrics play a key role, accounting for $10.3 \%$ of the overall star rating calculation in 2015. The degree to which these differences influence star ratings and bonus payments is unknown. The thresholds for the star rating cutpoints change each year and are based on data from previous years, meaning that even small changes in the proportion of adherence could move some health plans higher or lower when calculating a star rating. In view of this, further consideration and research for using pickup dates in place of adjudication dates is warranted; however, stakeholders, such as CMS, need to decide whether the magnitude of the differences in PDC are within an acceptable margin of error. In addition, the feasibility of having pharmacies report pickup dates to health plans or CMS would need to be considered, since this would require substantial investment in transmission and storage of a new data element. 


\section{Limitations}

This research has several limitations to consider. This study examined the impact of an automatic refill program from a small chain pharmacy in a midwestern state, and it is possible that the structure and impact of automatic refill programs may vary between pharmacy organizations and U.S. geographic regions. However, it is reasonable to predict that differences between adjudication dates and pickup dates will still exist, resulting in variations to the PDC calculation. The question of whether these differences in adherence would be clinically relevant is important. It is possible that reporting pickup date PDC in place of adjudication date PDC would not influence care for a majority of patients, but given the distribution of differences, it could be an important factor in determining if patients are adherent to their medications.

In addition, patients taking more than 1 study medication led to a PDC entry for each drug category into the analysis. This might have influenced the findings; however, adherence rates for different classes of medication have been documented in the literature. ${ }^{7}$ Another important limitation was the lack of random assignment to the automatic refill program. It may have been helpful to use a matching method, such as propensity score matching, to ensure the equivalence of the 2 groups, but the availability of appropriate patient characteristics was not available. As a result, it is not possible to suggest a causal relationship. ${ }^{18}$

\section{Conclusions}

This study provides evidence that the prescription pickup date, compared with the adjudication date, may be a better reflection of patient medication use and should be considered as an alternative way to measure medication adherence using PDC. Further investigation examining these differences in other community pharmacy and health plan settings is needed to confirm the findings in this study. The role of automatic refill programs in other community pharmacies needs to be considered given the decreased reliability of the PDC calculation that seems to occur. This study's findings demonstrate an opportunity to improve the accuracy of adherence measurement using claims data with the pickup date and can mean that the star ratings used by CMS are more accurate for all health plans.

\section{Authors}

COREY A. LESTER, PharmD, MS; KEVIN A. LOOK, PharmD, PhD; and MICHELLE A. CHUI, PharmD, PhD, Social and Administrative Sciences Division, University of Wisconsin-Madison School of Pharmarcy.

AUTHOR CORRESPONDENCE: Corey A. Lester, PharmD, MS, University of Wisconsin-Madison School of Pharmacy, 777 Highland Ave., Madison, WI 53705. Tel.: 608.262.0452; Fax: 608.262.5262;

E-mail: calester@wisc.edu.

\section{DISCLOSURES}

The project described was supported by the Clinical and Translational Science Award (CTSA) program through the NIH National Center for Advancing Translational Sciences (NCATS), grant UL1TR000427. The content is solely the responsibility of the authors and does not necessarily represent the official views of the NIH. Lester is employed as a pharmacist in the pharmacy chain that participated in this study. The authors report no other relevant conflict of interest.

Study concept and design were contributed primarily by Lester, along with Look and Chui. Lester took the lead in data collection, along with Look, and data interpretation, along with Look and Chui. The manuscript was written and revised primarily by Lester, along with Look and Chui.

\section{REFERENCES}

1. Academy of Managed Care Pharmacy, American Pharmacists Association. Medicare star ratings: stakeholder proceedings on community pharmacy and managed care partnerships in quality. J Am Pharm Assoc (2003). 2014;54(3):228-40.

2. Jacobson G, Neuman T, Damico A, Huang J. Medicare advantage plan star ratings and bonus payments in 2012. The Henry J. Kaiser Family Foundation. November 2011. Available at: https://kaiserfamilyfoundation. files.wordpress.com/2013/01/8257.pdf. Accessed September 7, 2016.

3. Reid RO, Deb P, Howell BL, Shrank WH. Association between Medicare Advantage plan star ratings and enrollment. JAMA. 2013;309(3):267-74.

4. Centers for Medicare \& Medicaid Services. Medicare Part C and D star ratings: update for 2016. August 5, 2015. Available at: https://www.cms. gov/Medicare/Prescription-Drug-Coverage/PrescriptionDrugCovGenIn/ Downloads/2016-Star-Ratings-User-Call-Slides-v2015_08_05.pdf. Accessed September 7, 2016.

5. Choudhry NK, Shrank WH, Levin RL, et al. Measuring concurrent adherence to multiple related medications. Am J Manag Care. 2009;15(7):457-64.

6. Nau DP. Proportion of days covered (PDC) as a preferred method of measuring medication adherence. Pharmacy Quality Alliance. 2012. Available at: http://www.pqaalliance.org/images/uploads/files/PQA\%20PDC\%20vs\%20 \%20MPR.pdf. Accessed September 7, 2016.

7. Yeaw J, Benner JS, Walt JG, Sian S, Smith DB. Comparing adherence and persistence across 6 chronic medication classes. J Manag Care Pharm 2009;15(9):728-40. Available at: http://www.jmcp.org/doi/pdf/10.18553/ jmcp.2009.15.9.728.

8. Coleman CI, Limone B, Sobieraj DM, et al. Dosing frequency and medication adherence in chronic disease. J Manag Care Pharm. 2012;18(7):527-39. Available at: http://www.jmcp.org/doi/abs/10.18553/jmcp.2012.18.7.527.

9. D'Souza AO, Smith MJ, Miller L-A, Doyle J, Ariely R. Persistence, adherence, and switch rates among extended-release and immediate-release overactive bladder medications in a regional managed care plan. J Manag Care Pharm. 2008;14(3):291-301. Available at: http://www.jmcp.org/doi/ abs/10.18553/jmcp.2008.14.3.291

10. Lester CA, Chui MA. The prescription pickup lag, an automatic prescription refill program, and pharmacy operations. J Am Pharm Assoc (2003). 2016;56(4):427-32

11. Morran C. Pharmacists confirm pressure from management to refill prescriptions automatically. consumerist. Consumerist. October 25, 2012. Available at: http://consumerist.com/2012/10/25/pharmacists-confirmpressure-from-management-to-refill-prescriptions-automatically/. Accessed September 7, 2016.

12. Matlin OS, Kymes SM, Averbukh A, et al. Community pharmacy automatic refill program improves adherence to maintenance therapy and reduces wasted medication. Am J Manag Care. 2015;21(11):785-91.

13. Benner JS, Glynn RJ, Mogun H, Neumann PJ, Weinstein MC, Avorn J Long-term persistence in use of statin therapy in elderly patients. JAMA. 2002;288(4):455-61. 
14. Krueger KP, Berger BA, Felkey B. Medication adherence and persistence: a comprehensive review. Adv Ther. 2005;22(4):313-56.

15. Centers for Medicare \& Medicaid Services. Medicare 2015 Part C \& D Star Rating Technical Notes. Draft. Updated September 3, 2014. Available at: https://www.cms.gov/Medicare/Prescription-Drug-Coverage/ PrescriptionDrugCovGenIn/Downloads/2015StarRatingsTechnicalNotes.pdf. Accessed September 7, 2016

16. Romano J, Kromrey JD, Coraggio J, Skowronek J. Appropriate statistics for ordinal level data: should we really be using t-test and Cohen's d for evaluating group differences on the NSSE and other surveys. Paper presented at: Annual Meeting of the Florida Association of Institutional Research; February 1-3, 2006; Cocoa Beach, FL. Available at: https://www.scribd. com/doc/45715000/Appropriate-Statistics-for-Ordinal-Level-Data. Accessed September 7, 2016.
17. Leech NL, Onwuegbuzie AJ. A call for greater use of nonparametric statistics. Paper presented at: Annual Meeting of the Mid-South Educational Research Association; November 7, 2002; Chattanooga, TN. Available at: http://files.eric.ed.gov/fulltext/ED471346.pdf. Accessed September 7, 2016. 18. Nichols A. Causal inference with observational data. Stata J. 2007;7(4):507. 